\title{
Correction to: Predation of treefrogs (Anura: Hylidae) with toxic skin secretions by the black lion tamarin (Leontopithecus chrysopygus, Callitrichinae)
}

\author{
Guilherme S. T. Garbino ${ }^{1}(1)$. Leonardo Henrique da Silva ${ }^{2,3,4} \cdot$ Rodrigo Gonçalves Amaral $^{4}$. \\ Gabriela Cabral Rezende ${ }^{3,4,5} \cdot$ Vinicius J. A. Pereira ${ }^{3} \cdot$ Laurence Culot $^{4}$
}

Published online: 18 May 2020

(C) Japan Monkey Centre and Springer Japan KK, part of Springer Nature 2020

\section{Correction to: Primates https://doi.org/10.1007/s10329-020-00818-1}

In the original publication of the article, the scientific name of veined treefrog should read as "Trachycephalus typhonius" instead of "Trachycephalus venulosus" according to recent taxonomic changes.

Publisher's Note Springer Nature remains neutral with regard to jurisdictional claims in published maps and institutional affiliations.

The original article can be found online at https://doi.org/10.1007/ s10329-020-00818-1.

Guilherme S. T. Garbino gstgarbino@hotmail.com

1 Programa de Pós-Graduação em Zoologia, Departamento de Zoologia, Instituto de Ciências Biológicas, Universidade Federal de Minas Gerais, Belo Horizonte, Minas Gerais, Brazil

2 Programa de Pós-Graduação em Ecologia e Biodiversidade, Departamento de Biodiversidade, Instituto de Biociências, Universidade Estadual Paulista, Rio Claro, São Paulo, Brazil

3 Black Lion Tamarin Conservation Program, IPÊ, Instituto de Pesquisas Ecológicas, Nazaré Paulista, São Paulo, Brazil

4 Laboratory of Primatology, Departamento Biodiversidade, Instituto de Biociências, Universidade Estadual Paulista, Rio Claro, São Paulo, Brazil

5 Programa de Pós-Graduação em Zoologia, Departamento de Biodiversidade, Instituto de Biociências, Universidade Estadual Paulista, Rio Claro, São Paulo, Brazil 